\title{
Vivir una experiencia fronteriza. Entre el aquí y el allí bajo un sistema totalitario en la obra de Albéna Dimitrova ${ }^{1}$
}

\author{
Ana Belén Soto ${ }^{2}$
}

Recibido: 15 de febrero de 2015 / Aceptado: 16 de julio de 2016

Resumen. En este artículo esbozaremos las huellas de la Historia del siglo XX en la construcción de la identidad nacional de esos países situados en esa Europa que llamábamos del Este. La Bulgaria de los años 80 se convierte en el escenario de una historia de amor prohibido y enfermizo que atraviesa las fronteras. Nous dînerons en français, la última novela de Albéna Dimitrova, se convierte así en el estandarte de una experiencia vivida teñida de ficción literaria y en el testigo privilegiado de una época marcada por las políticas totalitarias y la búsqueda identitaria.

Palabras clave: Francofonía; xenografías; identidad; totalitarismos; mujer; fronteras.

\section{[en] Live a border experience. Between the here and the elsewhere under a totalitarian system in the work of Albéna Dimitrova}

\begin{abstract}
In this article we will outline the traces of the History of the 20th century in the construction of the national identity of those countries located in that Europe that we called Eastern. The Bulgaria of the 80 s becomes the scene of a forbidden and sickly love story that crosses borders. Nous dinerons en français, the latest novel by Albéna Dimitrova, thus becomes the standard of a lived experience tinged with literary fiction and the privileged witness of an era marked by totalitarian policies and the search for identity.
\end{abstract}

Key words: Francophonie; xenography; identity; totalitarianism; woman; boundaries.

Sumario. Introducción. I. Albéna Dimitrova (sofia, 1967-). II. Frontières intra-muros. III. Frontière extra muros. Conclusión. Referencias bibliográficas.

Cómo citar: Soto, A.B. (2017). Vivir una experiencia fronteriza. Entre el aquí y el allí bajo un sistema totalitario en la obra de Albéna Dimitrova, en Revista de Filología Románica 34.2, 379-391.

Este artículo es el resultado de un trabajo de investigación sustentado Proyecto I+D+d FF2013-43483.

2 Dpto. de Filologías, Facultad de Formación de Profesorado de la UAM

anabelen.soto@uam.es 


\section{Introducción}

La frontière continue de constituer un élément essentiel de notre alphabet territorial. De la même façon que le géomètre se repère en fonction de points, lignes et aires, ces formes nous servent de repères dans notre expérience spatiale. Le géographe a également besoin de ces figures élémentaires pour décrire l'occupation humaine de notre planète, au risque de s'enfermer dans leur apparente simplicité. Réduire la frontière à une ligne tracée sur une carte n'est pas simplement un raccourci commode, c'est une démarche qui est à l'origine de l'invention de nos limites internationales: les frontières ont été inventées en même temps que les cartes (Amilhat Szary, 2015: 15).

El concepto de frontera como delimitación geográfica, en efecto, ha contribuido a situar a determinados colectivos de las sociedades sedentarias en un espacio concreto. Las mutaciones sociales, geográficas y políticas que han vivido las diferentes sociedades a lo largo de la Historia han puesto de manifiesto la movilidad y variabilidad que este concepto puede llegar a ofrecer al colectivo que en su territorio alberga, pues no son límites infranqueables. En efecto, en función de las guerras, los descubrimientos y las anexiones o desvinculaciones territoriales no sólo se ha ido dibujando la cartografía, sino que además han contribuido al desarrollo de la construcción nacional de los colectivos que allí se encuentran. Asimismo, respondiendo a las necesidades políticas, económicas y culturales de cada momento histórico, las fronteras han evolucionado hasta la creación de espacios abiertos en los que las personas y las mercancías pueden circular libremente, tal y como refleja el espacio Shenguen, y con ello se ha contribuido a la reestructuración social, cultural, familiar e identitaria en estos países.

Se inicia así un proceso de hibridación identitaria y lingüística en la que varios países en el mundo pueden usar un mismo idioma, ya sea como lengua materna, lengua oficial o como lengua de difusión cultural. En este contexto debemos poner de relieve el papel que juega la lengua francesa en el planeta, ya que tal y como se confirma en la página del Ministerio de Asuntos Exteriores francés, casi 119 millones de personas tienen el francés como idioma materno o de uso corriente, otros 63 millones de personas son francófonas parciales y 82 millones de jóvenes o adultos aprenden el francés, lo que suma un total de 264 millones de personas que comparten la lengua francesa. De tal manera que la amplitud del mosaico identitario que define el espacio de la Francofonía es un fiel reflejo los resultados de las transformaciones geopolíticas, de los intereses económicos y culturales e incluso del papel que juegan las políticas de promoción lingüística. El espacio de creación literaria francófono se convierte así en un testigo privilegiado de la proliferación de fenómenos que han contribuido a la evolución de una conciencia lingüística e identitaria desde un punto de vista artístico y literario. Las diferentes voces que escriben en el mosaico literario de la Francofonía han contribuido al cambio del paradigma nacional y ponen de manifiesto un concepto policromático de la sociedad en el que se persigue un enriquecimiento identitario bidireccional.

En la actualidad podemos observar cómo los flujos migratorios responden a impulsos tan diversos que pueden abarcar desde la necesidad de mejorar un estatus social y económico a través de la búsqueda de empleo, hasta la evacuación nacional 
por causas bélicas, pasando por aquellos aventureros que tan sólo pretenden disfrutar de una experiencia vital en otro país. El esquema de la migración presenta desde mediados del siglo pasado un cambio de paradigma y refleja el proceso de cambio que vive la mujer. En la actualidad, los datos indican que «plus de 200 millions de migrants dans le monde, dont la moitié de femmes» (Mazzella, 2014: 14), lo que determina un ámbito en el que la mujer también va adquiriendo cada vez mayor visibilidad.

La transgresión de las fronteras establecidas desde una perspectiva genérica se ha convertido a lo largo de las últimas décadas en una reivindicación que abarca todos los ámbitos de la sociedad. Así, desde un punto de vista artístico, la incorporación de textos escritos por mujeres en el espacio de creación literario representa el fin de la imposición del canon imperante hasta el momento y el enriquecimiento del panorama literario a través de la pluralidad de perspectivas, la incorporación de una sensibilidad inherente a la esencia femenina y la participación de personajes hasta el momento silenciados. Se trata, en efecto, de una contribución que beneficia a toda la sociedad con su reflexión, ya que:

la différence des sexes est une donnée fondamentale de l'expérience humaine et la vision qu'on a du monde et de la culture n'est pas la même selon que les femmes y apparaissent seulement en position d'objets, d'inspiratrices ou de lectrices, ou si elles figurent aussi en position de productrices et créatrices (Planté, 2003: 655668).

La transposición de los cuestionamientos sociales en el ámbito literario resulta una necesidad imperante que tiene cada vez más proyección. De ahí que no sólo surjan voces en femenino para romper esa frontera genérica en el ámbito literario, sino que además sean cada vez más numerosos los escritores que deciden legar su testimonio para contribuir en su labor de memoria histórica. En este contexto, hemos enfocado nuestro corpus de análisis en un marco cercano que nos ofrece una reflexión sobre la experiencia vivida en un sistema totalitario que marcó una nítida frontera en el corazón de Europa. Oana Orléa, Luba Jurgenson o Julia Kristeva son algunos nombres de mujer que siembran el panorama literario con sus textos marcados por el distanciamiento con su país de origen y la apuesta integradora por adoptar la lengua francesa como vehículo de expresión literaria. El carácter testimonial resulta inherente a la construcción literaria de estas mujeres que contribuyen a analizar la opresión ejercida por los sistemas totalitarios en el individuo.

En este amplio corpus, Albena Dimitrova representa un ejemplo paradigmático de la incorporación femenina en las xenografías presentes en el panorama literario francófono. Con la publicación en septiembre de 2015 de su obra Nous dînerons en français nuestra escritora toma la palabra para levantar la voz sobre una experiencia vivida teñida de ficción literaria. Alba y Guéo, sus protagonistas, guiarán al lector en una historia de amor entrelazada de Historia que se termina con la caída del sistema comunista en Bulgaria. Resulta imposible no establecer un paralelismo entre el nombre del personaje principal y la propia autora, de ahí que consideremos necesario empezar este análisis con la biografía de Albéna Dimitrova. Seguidamente analizaremos la dualidad vivida por el individuo bajo un sistema de tipo totalitario, revelando así las fronteras establecidas desde un punto de vista político en el ámbito social. Y, por último, esbozaremos el proceso de desterritorialización de la protagonista atra- 
vesando así una frontera geográfica que le permitirá transgredir todas las fronteras erigidas en el imaginario popular de una nación a través de la imposición totalitaria.

\section{Albéna Dimitrova (Sofia, 1967-)}

Escolarizada en el sistema soviético, la infancia de nuestra escritora se ha visto marcada por el yugo de un sistema totalitario. Las largas filas de espera en los supermercados, la escasez de productos de primera necesidad, la colectivización de las tierras o la abolición de la propiedad privada son algunas de las características más conocidas de esta época en los países de esa otra Europa situada al Este. Se suele obviar, no obstante, una característica esencial en el proceso de anexión gubernamental: la incorporación de aquellos países antaño denominados satélites de la URSS; ésta se basa en un proceso impositorio en el que no sólo se implanta un sistema político y económico, sino que además se aplica en el ámbito social e identitario con la creación de un aparato político de delación, sanción e intimidación basado en el miedo. De esta manera, todos aquellos individuos que discrepaban con las medidas gubernamentales impuestas por Moscú tenían que elegir entre alzar su voz y acabar viviendo una experiencia concentracionaria o silenciar sus pensamientos y sufrir una alienación verbal. En este contexto, numerosos eran aquellos que abogaban por la subordinación, aunque tuviesen que usar un doble discurso que, para Tveztan Todorov supone un proceso similar al que puede vivir una persona que habla dos idiomas, pudiendo sin embargo distinguirse:

par leur vocabulaire, un peu par leur syntaxe, mais surtout par le principe de leur fonctionnement. Le discours privé peut être régit par plusieurs exigences: ainsi par la recherche du plaisir de l'interlocuteur; ou encore par ce qu'on pourrait appeler la vérité d'adéquation, les paroles énoncées devant décrire le monde ou exprimer les opinions du sujet de la manière la plus exacte possible. Le discours public, lui, ne se soucie que d'une «vérité» de conformité: pour être jugée, la parole n'est pas confrontée àla réalité empirique, pas plus qu'on ne s'interroge sur le plaisir éventuel du partenaire; l'unique exigence est qu'elle soit conforme à d'autres discours déjà existant et connus de tous, à une opinion juste sur toute chose (Todorov, 1996: 42).

De tal manera que el individuo se ve confrontado a una pluralidad discursiva que, para el intelectual sofiota, desemboca en una «schizofrénie (si l'on veut garder à ce terme sa signification commune de scission de la personnalité, d'incohérence mentale, et son association à une forme de détresse)» (Todorov, 1996: 44).

En este contexto de imposición y presión crece Albéna Dimitrova, una joven que recuerda sus primeros años con la candidez de una infancia en la que podía disfrutar de las calles de Sofía cubiertas de nieve en invierno con sus padres y de la vegetación de los Balcanes en verano, gracias a que pasaba sus veranos en el pueblo de sus abuelos. Durante esos años, Albéna Dimitrova recuerda, cómo dentro de los límites impuestos por el sistema, la despreocupación propia de la adolescencia les permitía divertirse transgrediendo los códigos impuestos con cosas tan simples como coser de rojo los botones de su uniforme azul. Una época en la que Albéna Dimitrova sintió 
despertar en ella la necesidad de ver más allá de sus fronteras e intentó que la aceptaran en la Universidad británica de Oxford. No obstante, su proyecto de estudios en Inglaterra se vio truncado y se vio obligada a aceptar una beca para estudiar en Francia, lo que supuso iniciarse al aprendizaje del francés durante el verano y poder así empezar sus estudios en la Sorbonne.

A nuestra autora le gusta recordar que cuando llega a París, unos meses antes de la caída del comunismo, sólo sabía decir: bonjour y voyage. Dos palabras cargadas de significado, porque gracias a su viaje iniciático en Francia, Albéna Dimitrova podrá saludar al mundo libre. Su aprendizaje del francés se hace de manera paulatina y, de hecho, recuerda con cariño en una entrevista dada a France Inter aquellas clases de Historia de la Economía que seguía con dificultad y de la suerte que había tenido como economista al poder abrazar su nueva lengua, el francés, con esa lengua internacional de las matemáticas. Tras unos años, Albéna Dimitrova obtendrá su Licenciatura en Económicas, una formación que le permitirá investigar sobre la modernización matemática de los riesgos sistemáticos aplicados a la privatización de los países del este y a los mecanismos de la corrupción. Un campo de investigación profundamente marcado, de nuevo, por su experiencia vivida, ya que ella misma recuerda con tristeza aquellos primeros años de la transición en la que su familia lo había perdido todo y era ella, una estudiante becada por el Ministerio, la que tenía que ayudar a su familia enviándoles comida.

Por otra parte, debemos poner de relieve en su trayectoria profesional que Albéna Dimitrova ha sido socia fundadora y Secretaria General de la Alianza Francia-Bulgaria bajo la presidencia de Pierre Consigny. Una asociación que persigue una triple misión: por una parte, pretende vincular los países que son embajadores en los ámbitos político, económico, social y cultural; asimismo, quiere acompañar la transformación y adaptación búlgara para su integración en la Unión Europea; y, por último, trabaja para establecer lazos de cooperación entre las sociedades civiles. Con todo, a partir de 2006, Albéna Dimitroba decide consagrarse íntegramente al mundo artístico y literario, escribiendo novelas y dirigiendo programaciones artísticas, de las que debemos resaltar el Festival de Danza Contemporánea de Sofía (SDW) y el Festival de Teatro ARTA en Bulgaria central.

Con este telón profesional de fondo, nuestra autora va tejiendo su historia como un puente entre las dos naciones que conforman su nueva identidad. Una identidad múltiple, al igual que la sociedad plural que la acoge en la que se crea un nuevo paradigma intercultural y multilingüe. Se trata, en efecto, de un nuevo perfil de ciudadano que contribuye a la modificación de los cánones estéticos nacionales. Con la publicación de Goût bulgare, portrait de femmes en Bulgarie 3 (2008), Méditance ${ }^{4}$ (2009) y Nous dînerons en français (2015) ${ }^{5}$, nuestra autora pone de manifiesto tanto

Utilizando como pretexto la vida cotidiana de cinco mujeres, Albéna Dimitrova presenta una novela de marcado carácter sociológico en la que se representa una mujer fuerte, libre y plural, capaz de reinventarse. Paralelamente, encontramos una referencia a esas mujeres búlgaras que atravesaron las fronteras para instalarse en París y vivir con éxito su ideal de existencia. Julia Kristeva, Nadège Ragaru o Darina Krastinova son algunos de los nombres que aparecen. Por ello, podemos afirmar que se trata de una novela de carácter sociológico en la que se pone de relieve el papel de la mujer, tanto a nivel personal como profesional, antaño silenciado por los paradigmas nacionales imperantes.

$4 \quad$ La lengua alcanza su máximo protagonismo en esta obra poética. Se trata de un canto en defensa de la escritura en el que no duda en usar palabras arcaicas o en desuso como muestra de riqueza lingüística.

5 Las referencias musicales y gastronómicas se entrelazan con una exquisita historia de amor frustrado en un con- 
su dominio de la lengua francesa como el valor testimonial de sus novelas, porque «le passé ne demande pas seulement d'être connu avec précision, il comporte aussi une leçon pour le présent» (Todorov, 2009: 223). Por ello, podemos afirmar que Albéna Dimitrova se convierte en un ejemplo paradigmático de una generación profundamente marcada por los cambios políticos de 1989 y que

marquent une autre étape dans la recherche et dans la construction d'une nouvelle identité culturelle et artistique [...], qui réfléchissent sur le totalitarisme et le dénoncent [...] [et qui] met l'accent sur la question, «Qui sommes-nous?» en scrutant le passé et le présent. Notamment «Nous»- les gens de l'Est de l'Europe, les Européens, les citoyens du monde? (Enderlein y Mihova, 2013: 18).

De tal manera que el proceso de escritura se convierte en un puente que se edifica sobre un nuevo lenguaje para construir el reflejo de la memoria.

\section{Fronteras intra-muros}

Nous dînerons en français es una obra que evoluciona en la frontera de la ficción y de la realidad, de lo imaginario y de lo vivido, del recuerdo y de lo escuchado que empieza así:

Dès qu'on s'éclipsait avec Guéo, notre vigilance se réduisait, et de jour en jour classait autrement l'ordre de repères. L'ombre était claire, transparente, il n'y avait rien à surveiller, et ce rien à surveiller appelait toutes les surveillances. Les services secrets guettaient. Les Russes, dépêchés par sa femme, la gentille fille du général en chef des forces de terre de l'Union soviétique, les Syriens, les Yémenites, ses collègues du Politburo et, bientôt, même les amis, les réformateurs (Dimitrova, 2015: 11).

El contexto está claro y los personajes delimitados por la frágil línea de un amor prohibido entre un hombre casado, con la hija de un militar que ejerce un alto cargo en el gobierno, y la mujer que toma la palabra.

En este discurso existe una dicotomía aparente entre la transparencia y la opacidad que marcará la historia de este amor nacido en el ocaso del sistema comunista. El preludio narrativo viene marcado por una reflexión escrita en cursiva en la que la protagonista se sitúa en el trágico final, de tal manera que el edificio narrativo se construye con los vaivenes de la memoria a modo de flashback. Todo había comenzado en el hospital gubernamental de Sofía, donde Alba había sido transferida algunos días antes que Guéo. Este lugar representará no sólo el punto de partida de la historia de amor, sino que también marcará el inicio de un cuestionamiento político e identitario, que proseguirá al hilo de las páginas.

Cuando aparece un hospital aparece en el imaginario colectivo un lugar destinado a los enfermos, a todas aquellas personas que sufren alguna enfermedad y que, por

texto no menos frustrado: los últimos años de las políticas totalitarias en el este de Europa. La novela empieza en ese punto y final de una Historia que deberá ser recordada para ser vivida, que tendrá que ser añorada para no ser olvidada y que finalmente se quedará en una promesa por cumplir. 
consiguiente, necesitan ser curados. La enfermedad se erige, pues, en una suerte de personaje omnipresente estableciendo así la primera frontera entre una sociedad enferma y una sociedad sana. Entre los muros del hospital gubernamental se establece, a su vez, una diferencia clara entre los miembros del Partido en función de su grado, rango y categoría. De tal manera que en esta primera zona afectada se pone de manifiesto el cuestionamiento de la esencia misma del comunismo: la abolición de las clases sociales. En efecto, la ideología comunista afirma que las sociedades deben presentarse como un conjunto homogéneo indiferenciable por clases o castas. No obstante, mientras la joven Alba se encontraba en el hospital pudo comprobar que sí que existía un elemento diferenciador:

les étages étaient leur force. Les dirigeants de la République du peuple régissaient tout par les étages. Les logements aussi étaient distribués selon d'indivisibles consignes d'étage. On y déchiffrait les grades et les rangs, les promotions et les descentes aux enfers. Les dirigeants partageaient leurs immeubles avec des ouvriers méritants. Ils se devaient de rester en contact avec le peuple, mais pas au même étage (Dimitrova, 2015: 36).

Con esta observación, la protagonista pone en tela de juicio un principio de igualdad abogado por un sistema que, finalmente, se rige por atribuciones políticas de derechos y privilegios. De tal manera que esos privilegios se veían reflejados en materia de sanidad, de educación, de vivienda e incluso en los quehaceres cotidianos.

En efecto, el simple hecho de comer exhibía esas diferencias, pues tal y como recuerda Alba:

À l'étage du Politburo, les repas étaient servis dans une vaste salle à manger. Les lustres étaient en cristal, nos assiettes en porcelaine fine, les chaises ne grinçaient pas.

Nos plats étaient chapeautés d'élégants couvercles pour les tenir au chaud. Astiqué jusqu'à la brillance, leur métal reflétait les moindres traits. [...] Les convives discutaient pesamment, ne doutant pas un seul instant que leur existence ait une importance pour la bonne marche du monde. Leur présence ici signifiait bien qu'ils souffraient, mais les soins qu'ils recevaient à l'hôpital du gouvernement me donnaient l'impression de côtoyer une espèce rare dont la santé était vitale pour nous tous (Dimitrova, 2015: 37).

Se trata de una observación que presenta la fuerza misma del sistema, ya que para no desaparecer había que cuidar, tratar y sanar a esos dignatarios que eran deificados por el Partido con el único objetivo de asegurar la pervivencia del sistema.

La opulencia de la que disfrutaban los líderes del aparato estatal choca, sin embargo, con la realidad latente en la sociedad búlgara del momento. El descontento social estaba intrínsecamente ligado a los problemas de abastecimiento de los supermercados y a la obtención de productos básicos y de primera necesidad. Los carnets de racionamiento y las largas colas en los supermercados se convertían en una realidad cotidiana denunciada por muchos coetáneos de Albéna Dimitrova. De ahí que intelectuales como Todorov hayan llegado a criticar la emergencia de un nuevo sistema de castas con una jerarquía mucho más compleja que la establecida por el sistema de clases sociales, pues «être membre du Parti ne vous avance pas 
encore à grand-chose, on peut ensuite progresser vers le Comité central, puis vers le Bureau politique (membres suppléants et membres titulaires, secrétaires et vice-secrétaires)» (Todorov, 1996: 38). La única diferencia existente entre las dos maneras de jerarquizar la sociedad se establece, según Todorov, en la versatilidad de los escalones de este nuevo sistema social, ya que se podía subir y bajar según los designios de los superiores. Por ello, podemos afirmar que se atisba en estas sociedades una esfera competitiva que despierta las ambiciones personales.

La primera frontera se establece, por consiguiente, en la manera en la que el aparato estatal se define frente al conjunto de la sociedad. Alba pertenece a esa esfera social alejada de los dirigentes gubernamentales, pero presenta un caso de estudio interesante para los médicos y, por ello, fue trasladada al hospital gubernamental. Afectada por una parálisis del tronco superior con tan sólo 16 años, los médicos se debatían entre la amputación o los posibles métodos que permitirían curar a la protagonista. Guéo también tiene una suerte de parálisis, pues presenta un cuadro de disfunción eréctil. Por ello, podemos afirmar que la parálisis vivida por los protagonistas simboliza una prolongación de la parálisis vivida por un sistema marcado por el estancamiento político, social y cultural y por la proliferación de un descontento generalizado.

En este contexto, los personajes viven una historia de amor y pasión en la que los papeles tradicionales del hombre y de la mujer se ven transgredidos. En efecto, Alba se presenta como una joven fuerte, autónoma y con un marcado carácter sexual que establece una relación de igual a igual con Guéo, a pesar de la diferencia de edad. Un ejemplo de ello se establece en el recuerdo de su primera relación sexual:

Nos conversations débordaient sur tous les terrains et arrivaient jusqu'aux confins incertains des relations amoureuses. Des amours en général, des amours en particulier.

[...] Je lui avais fait croire que j'étais vierge. Cela m'avait semblé important, nécessaire. Lui, il ne demandait rien. Je ne sais quoi dans son regard avait mué en moi ce mensonge en vérité. Je sentais la présence d'une chair ignorée, d'un appel à déflorer les cloisons inexplorées. Une virginité perdre sans perte, déjouer sa stérilité sans les réprimandes de la pureté (Dimitrova, 2015: 45).

Con estas palabras podemos observar que se trata de una mujer plenamente consciente de su apetito sexual y de su derecho al ejercicio del placer con la persona a la que ama. La parálisis y lo prohibido no frustra las relaciones que mantienen los dos personajes y Alba se queda embarazada, unos meses antes de cumplir sus 18 años. Ante la situación Guéo, alto dirigente del Partido, mueve los hilos para que Alba pueda abortar en un entorno silenciado por el poder. Ella, no obstante, se resigna a someterse a Guéo y a la intrusión gubernamental en su vida privada y decide esperar hasta sus 18 años para abortar en una clínica pública, a pesar de encontrarse en ese límite permitido. Con esta decisión esta joven pone de manifiesto la emergencia de un nuevo modelo de mujer que lucha por alzar su voz en un momento en el que «le communisme avait bien aboli la propriété des terres mais sa défaite était peut-être d'avoir cherché s'approprier les cœurs» (Dimitrova, 2015: 87). La intrusión gubernamental en la esfera privada se convierte así en el talón de Aquiles de un sistema en el que mujeres como Alba atraviesan la frontera de los prohibido y, a pesar de su parálisis, dan un paso firme hacia la construcción de su propio futuro erigiéndose en 
motor de esa modernidad cambiante en la que la mujer aparece como una mujer para el hombre y no como la mujer del hombre.

Podemos afirmar a través de esta reflexión sobre la mujer en las sociedades totalitaria que Albéna Dimitrova presenta otra transgresión fronteriza. He aquí un tema que ya analizó en su primera novela Goût bulgare, portrait des femmes en Bulgarie y en la que plasma con total claridad una reflexión despojada de florituras y llena de pasión. Para nuestra autora,

Dès 1948, le sexe fut supprimé des catégories socio-professionnelles. À travail égal, salaire égal. Depuis les murs aux grandes affiches de propagande, ouvriers et ouvrières-modèles nous saluaient à la taille égale, épaules contre épaules, pionniers et pionnières la main dans la main, pas un brin qui dépasse. Mot d'ordre: nous sommes égaux! Dans les universités afflua une mixité effervescente, inattendue, débordante. À défaut de révolution sexuelle, nous avons connu l'abolition sexuelle (Dimitrova, 2008: 7).

En este fragmento situado a modo de introducción de esta novela con marcado carácter sociológico, la autora pone de manifiesto su crítica ante el papel que desempeña la mujer en los regímenes totalitarios. En efecto, las necesidades de mano de obra hicieron que el trabajo de la mujer se equiparase al de los hombres, que las mujeres se formasen para que pudiesen tejer el engranaje del ámbito profesional necesario y que siguiesen los cauces marcados por el Partido. Mientras tanto, en esa Europa Occidental de los años 60 y 70 las mujeres se levantaron para perseguir una emancipación sexual y la afirmación de la igualdad de género. Se trata de una revolución que se acompaña de una serie de medidas que permiten que las mujeres accedan a una igualdad legislativa tanto en el ámbito personal como profesional. De este modo, las mujeres irán consiguiendo paulatinamente la igualdad en el seno de sus matrimonios, el derecho a la contracepción y el derecho al aborto, entre otras medidas. En el caso de Bulgaria, al igual que otros muchos países situados en la Europa del Este, esa revolución se convirtió en una imposición estatal, por lo que para Albéna Dimitrova representa la amputación de una parte del proceso, la ablación de la toma de conciencia de la feminidad y, de ahí, que la imposición provoque una abolición sexual.

La lengua elegida para este canto a la feminidad y a la liberación del yugo de los totalitarismos es el francés, ese idioma del que se enamoró y que se ha convertido en su patria de adopción. Adoptando su lengua de acogida como vehículo de expresión literaria Albéna Dimitrova se convierte así en una de esas pocas escritoras que no dudan en escribir en su francés-cirílico, o con acento como le gusta decir a ella, porque «[elle]s'est sentie "chez elle" dans l'ailleurs, [et] a choisi, sans problème et sans complexe, d'écrire dans une autre langue» (Mihova, 2013: 224).

\section{Frontera extra muros}

El tiempo estimado para el tratamiento de Guéo llegaba a su fin y Alba de manera paulatina iba ganando confianza en sus piernas y venciendo así su enfermedad. Esta historia de amor que surgió bajo el signo de la enfermedad, consiguió sobrevivir fuera de estas fronteras, aunque no sin altibajos. Guéo seguía tristemente casado en 
segundas nupcias con la gentille fille du général y Alba tenía que terminar sus estudios en el instituto. Por ello, vivían su historia de amor en secreto y con los vaivenes marcados por la alienación mental que provoca la creación de un discurso y de un lenguaje corporal que se usa en la esfera pública y la pasión con la que ese mismo lenguaje opera en la vida privada. Su engranaje lingüístico constituye el único medio para resistir y escapar de la opresión y será en este contexto en el que Guéo decida enseñar francés a Alba, porque Francia será el lugar elegido por Guéo para ponerla a salvo.

La elección de París no es un lugar anodino, sino que se trata del lugar en el que Albéna Dimitrova vive su propia experiencia de exilio; donde, además, al igual que su creación, es conocedora de la caída del Muro de Berlín y del inicio de la transición democrática en su país. París, además, representa en el imaginario colectivo el centro neurálgico de un país que, para muchos, ha sido el centro del mundo intelectual y político en los últimos siglos. Un país que amamantó los ideales de la Revolución Francesa, que desarrolló el espíritu de la Declaración de los Derechos Humanos y que también representa el corazón de un vasto imperio colonial vigente hasta hace algo menos de un siglo. El lugar elegido, por consiguiente, simboliza el ideal de una vida en un país democrático en libertad y será por ello por lo que las primeras palabras de esta novela presenten una reflexión sobre ese proceso de adopción de una lengua y una cultura que con el tiempo abrazará cómo propias:

«Parlait-il vraiment français, le parlait-il bien?

- Vraiment, je crois...»

Comment vérifier et croire à la fois? Je n'ai jamais pu échanger un mot dans cette langue avec lui. Nous nous étions pourtant donné rendez-vous à Paris pour un premier dîner en français (Dimitrova, 2015: 9).

La lengua se convierte así en un lazo que unirá para siempre a estos dos amantes a pesar de la distancia.

El primer contacto que tuvo Alba con el francés fue a través de un regalo de la parte de Guéo. Se trataba de un diccionario muy especial, «le sien, son tout premier, celui qui l'avait suivi depuis la Serbie. Il avait appartenu à quelqu'un d'autre avant lui, le nom y figure toujours inscrit en rouge d'une écriture appliquée» (Dimitrova, 2015: 131). Un diccionario en desuso, pero que con ella comenzaría un nuevo viaje. En el pacto literario el viaje y la lengua adquieren un carácter cada vez más importante, pues Guéo fue un hijo del comunismo «ramassé dans la rue à l'âge de onze ans» (Dimitrova, 2015: 25) y educado para formar parte de la élite política del país para dirigir el sistema con un sentimiento de lealtad propio al agradecimiento del desamparado. Guéo sabe que él no puede escapar de un sistema que le rescató de la miseria y que le dio poder y prestigio, pero no quiere que Alba viva los daños colaterales de sus propias decisiones. La única vía de escape para Alba es la fuga, el desaparecer, el atravesar la frontera sin mirar atrás. De tal manera que el francés se convierte para estos amantes en la lengua de la transgresión y de la desobediencia, del amor y del cariño, de la protección y de la liberación.

Una vez instalada en París, Alba pasa horas y horas repasando las reglas gramaticales, aprendiendo nuevas palabras y viendo otras realidades. Con el objetivo de tener una cena en un francés perfecto, Alba recuerda cómo: 
Je veillais sur une lecture des règles de la concordance de temps. Je m'attardais sur les temps passés, si nombreux, des simples, des composés, des imparfaits, des plus-que-parfaits, des futurs et déjà antérieurs. Les verbes réguliers et les irréguliers défilaient sous mes yeux. Je photographiais leurs actions et leurs sonorités, comme dans notre jeu avec Guéo (Dimitrova, 2015: 9).

Se trata, no obstante, de un sueño truncado por una llamada en la que anuncian cómo tras la caída del Muro de Berlín: «Il s'est donné la mort, He killed himself; Cette nuit, dans son bureau, à l'appartement de Sofia, This night, at his place, at Sofia; He killed himself, He is not coming, he will never come, Il ne vient pas, il ne reviendra jamais, il s'est donné la mort» (Dimitrova, 2015: 178). Se trata de un trágico acontecimiento que deja a Alba «vidée de toutes [ses] langues, pas un mot, pas un bruit. Fantôme errant dans le silence» (Dimitrova, 2015: 178).

Paralelamente, mientras Alba mimaba con cariño todo aquel aprendizaje lingüístico y cultural, Guéo se había propuesto terminar la misión que le habían asignado sus amigos los reformadores: redactar un dossier que promulgase el saneamiento del sistema y preparase una transición hacia nuevas vías gubernamentales. Con su publicación Guéo comete un doble asesinato: por una parte, un parricidio, ya que será uno de los hijos del sistema el que acabe con él, y, por otra parte, su propio suicidio, ya que es plenamente consciente que los actos llevados a cabo para beneficiar el buen funcionamiento del sistema saldrán a la luz. Guéo deja, no obstante, una semilla de esperanza a su paso: Alba, pues al igual que él fue rescatado por el comunismo, Guéo rescatará a esta joven enérgica y dinámica de las consecuencias directas e indirectas infligidas a la sociedad tras la caída del sistema.

Alba, seguirá esperando en vano esa cena en francés que nunca llegará, mientras va rehaciendo su vida integrándose en la sociedad de acogida. En París seguirá frecuentando uno de los amigos de Guéo, terminará sus estudios universitarios y conseguirá, al igual que su creadora, forjar su construcción identitaria poniendo de manifiesto su doble identidad lingüística y cultural. Albéna sitúa a Alba en un puesto significativo, «à l'Assemblée nationale à Paris [...] [comme] "chargée des PECO et de la Russie» (Dimitrova, 2015: 186) en el que pueda poner al servicio de la reestructuración nacional su conocimiento identitario profundamente influido por los fenómenos de desterritorialización y de reterritorialización.

\section{Conclusión}

Para terminar y a modo de conclusión podemos afirmar que la obra de Albéna Dimitrova representa un ejemplo paradigmático de este mosaico literario escrito por mujeres que vienen de esa otra Europa que se llamó durante varias décadas «del Este». Sus palabras conforman el edificio principal de una historia ficcional cargado de testimonio, marcado por el desarraigo identitario y afectado por el dolor de la guerra. Estos puntos se convierten en los topoi de una literatura en la que la imbricación histórica resulta indisociable de la construcción identitaria. Para Stéphane Altasserre se trata de una escritora que ha sabido abrazar su doble pertenencia cultural y lingüística, pues afirmar: 
Si elle est écrivaine d'origine bulgare, elle reste, avant tout, une auteure française: sa création littéraire et ses qualités de médiatrice de l'activité artistique son inhérentes à l'usage du français comme langue privilégiée d'expression. Sa bulgarité lui offre, pourtant, une ouverture d'esprit génératrice de création (Enderlein y Mihova, 2013: 178).

En efecto, Albéna Dimitrova pertenece a ese grupo reducido de escritoras búlgaras que han conseguido hacerse un hueco en el espacio de creación literario francés.

Por otra parte, resulta interesante observar cómo, con la caída del Muro de Berlín, la hasta entonces Unión Soviética desaparece al igual que sus países antaño satélites. Se convierten en nuevas naciones, con nuevas realidades y nuevas leyes, lo que implica que una parte de la identidad de origen se relega a la casilla de la memoria. Esta circunstancia ha llevado a un buen número de migrantes a forjarse una nueva identidad también de partida. En este cambio identitario, ¿dónde podemos situar la elección de un país y de una lengua como tierra de acogida y vehículo de expresión literaria? ¿Podríamos afirmar que con el desmembramiento de la antigua URSS sus ciudadanos se sienten más cercanos a la interculturalidad? Lejos de tener una respuesta concreta y universal, aún nos encontramos en el estado de la investigación y es por ello por lo que nos permitimos hablar y hacer hablar a esas voces francófonas que vienen de esa otra Europa que llamábamos del este. Un abanico literario que, para Joanna Nowicki y Catharine Mayaux sigue estando

Marginalisée, souvent occultée au profit de la francophonie du Sud ou d'autres francophonies mieux reconnues dans une géopolitique mondiale comme dans les champs d'études universitaires, elle s'exprime pourtant à travers la littérature, l'art, la philosophie, l'histoire, [etc]. Se pencher sur l'Autre Francophonie consiste aussi à remettre en valeur un aspect important de la culture européenne, à savoir le lien entre les diverses parties de l'Europe, notamment celles que l'histoire a cruellement séparées, lien créé par des valeurs communes, une sensibilité commune, une vision du monde commune, un art de vivre partagé (Nowocki y Mayaux, 2012: 9).

\section{Referencias bibliográficas}

Albert, Christiane (2005): L'immigration dans le roman francophone contemporain. París: Karthala.

Alfaro, Margarita (2009): Interculturalidad y creación artística. Espacios poéticos para una nueva Europa. Madrid: Calambur.

Alfaro, Margarita et alii (2000): La francophonie: enjeux et identités, Actas del Congreso organizado por la Universidad Autónoma de Madrid, Madrid.

Amilhat Szary, Anne-Laure (2015): Qu'est-ce qu'une frontière aujourd'hui. París: PUF.

Enderlein, Évelyne y Mihova, Lidiya (2013): Écrire ailleurs au féminin dans le monde slave au XXe siècle. París: L'Harmattan.

Dimitrova, Albena (2008): Goût Bulgare, portraits de femmes en Bulgarie. Monaco: du Rocher.

Dimitrova, Albena (2015): Nous dînerons en français. París: Galaade.

Kristeva, Julia (1991): Étrangers à nous-même. París: Flammarion. 
Mazzella, Sylvie (2014): Sociologie des migrations. París: PUF.

Nowocki, Joanna y Mayaux, Catherine (2012): L'autre francophonie. París: HonoréChampion.

Planté, Christine, (2003): "La place des femmes dans l'histoire littéraire: annexe, ou point de départ d'une relecture critique?", en Revue d'histoire littéraire de la France 3/2003 (Vol. 103), p. 655-668. https://www.cairn.info/revue-d-histoire-litteraire-de-la-france-2003-3-page-655.htm

Porra, Véronique (2001): Langue française, langue d'adoption. Une littérature "invitée" entre création, stratégies et contraintes (1946-2000. Olms: Hildescheim.

Stantcheva, Roumiana (2008): "Les Études Balkaniques et la Francophonie roumaine et bulgare actuelle", en Moussakova, S., Les cahiers européenns de la Sorbonne nouvelle, Nouveaux visages de la Francophonie en Europe. París: Bruylant, pp. 171-182.

Todorov, Tveztan (2009): L'expérience totalitaire. París: du Seuil.

Todorov, Tveztan (1996): L’homme dépaysé. París: du Seuil. 